NASA Technical Memorandum 102135

AIAA-89-2793

\title{
A Numerical Study of Chemically Reacting Flow in Nozzles
}

Thomas J. VanOverbeke

National Aeronautics and Space Administration

Lewis Research Center

Cleveland, Ohio

and

Jian-Shun Shuen

Sverdrup Technology, Inc.

NASA Lewis Research Center Group

Cleveland, Ohio

Prepared for the

25th Joint Propulsion Conference

cosponsored by the AIAA, ASME, SAE, and ASEE

Monterey, California, July 10-12, 1989

\section{N/SA}




\title{
A NUMERICAL STUDY OF CHEMICALLY REACTING FLOW IN NOZZLES
}

\author{
Thomas J. VanOverbeke \\ National Aeronautics and Space Administration \\ Lewis Research Center \\ Cleveland, Ohio 44135
}

and

\author{
Jian-Shun Shuen \\ Sverdrup Technology, Inc. \\ NASA Lewis Research Center Group \\ Cleveland, Ohio 44135
}

\begin{abstract}
The Space-Station uses small rocket motors, called thrusters, for orientation control. Because of the lack of viable design tools for small rockets, the initial thruster design was basically a very small version of a large rocket motor. Thrust measurements of the initial design were lower then predicted. To improve predictions it was decided to develop a version of the RPLUS2D reacting flow code for thruster calculations. RPLUS2D employs an implicit finite volume, lower-upper symmetric successive overrelaxation (LU-SSOR) scheme for solving the complete two-dimensional Navier-Stokes equations and species transport equations in a coupled and very efficient manner. The combustion processes are modeled by a 9-species, 18 step finite-rate chemistry model, and the turbulence is simulated by a Baldwin-Lomax algebraic model. The code in this work is extended to handle multiple subsonic inlet conditions where the total mass flow is governed by conditions calculated at the thruster-throat. Results are shown for a thruster design where the overall mixture ratio is hydrogen rich. A calculation of a large area ratio divergent nozzle is also presented.
\end{abstract}

\section{INTRODUCTION}

For the space station relatively small rocket engines, called thrusters, are needed for attitude control. Using current successful designs for large rockets and the TDK code (which is based on the method of characteristics) a thruster was designed, built and tested. Thrust measurements of the small rocket motor were about 10 percent lower than predicted. As the thrusters may be expected to operate for long periods of time this error can have a large effect. In addition, it is very costly and time-consuming to design, fabricate, and test rocket engines. To cut down on costs more accurate predictions are needed. Several sources of error were identified. First, the TDK code does not predict the boundary layer as accurately as needed for thrusters. The TDK code calculates the flowfield in two passes. In the first pass, no boundary layer is calculated, then the flow is corrected for a boundary layer in the second pass. The boundary layer has a much larger effect in the rather small thruster, so calculating the boundary layer in this manner is not accurate enough. Secondly, the TDK code separates the flow into separate flowstreams. Unless a large number of flowstreams is specified, no mixing is allowed between flowstreams. This causes a large demand on computer resources. 
Finally, the TOK code uses an equilibrium chemistry model, which will tend to overpredict mixing and the extent of chemical reactions. Rather than adjust the constants in the TDK code to correctly predict the flow fields in thrusters, it was decided to develop a more efficient modern code. After a period of study, the RPLUS2D code was chosen for development. RPLUS2D has previously been developed for the study of mixing and chemical reactions in flow fields of ramjets and scramjets.

RPLUS2D employs an implicit finite volume, lower-upper symmetric overrelaxation (LU-SSOR) scheme for solving the complete two-dimensional NavierStokes equations and species transport equations in a coupled manner, with real gas properties. The LU-SSOR technique was originally proposed by Jameson and Turkel (ref. 1) and developed by Yoon and Jameson (ref. 2). The code was extended to treat chemically reacting flows by Shuen and Yoon (ref. 3 ). The LU-SSOR scheme treats the inviscid flux and chemical source terms implicitly, but the viscous term explicitly. Despite being implicit, the LU scheme requires only scalar inversions for nonreacting flows and block-diagonal inversion for reacting flows, which saves considerable CPU time without using a diagonalization procedure. Furthermore, because of the special structure of the left-hand side (LHS) LU operators in the LU-SSOR scheme, the two L and $U$ operators can be fully vectorized while the LHS operators in most other implicit schemes are not fully vectorizable.

\section{GOVERNING EQUATIONS}

The two-dimensional Navier Stokes and species transport equations for a chemically reacting flow with $\mathrm{N}_{S}$ species, in Cartesian coordinates, are given by equations (1) and (2).

$$
\frac{\partial W}{\partial t}+\frac{\partial(F-F v)}{\partial x}+\frac{\partial\left(G-G_{v}\right)}{\partial y}=S
$$

where

$$
\begin{aligned}
& \mathrm{W}=\left|\begin{array}{l}
\rho \\
\rho \mathrm{u} \\
\rho \mathrm{v} \\
\rho \mathrm{E} \\
\rho \mathrm{Y}_{\mathrm{i}}
\end{array}\right|, \quad \mathrm{F}=\left|\begin{array}{l}
\rho \mathrm{u} \\
\rho \mathrm{u}^{2}+\mathrm{P} \\
\rho \mathrm{uv} \\
\mathrm{u}(\rho \mathrm{E}+\mathrm{P}) \\
\rho \mathrm{uY}
\end{array}\right|, \mathrm{G}=\left|\begin{array}{l}
\rho \mathrm{v} \\
\rho \mathrm{uv} \\
\rho \mathrm{v}^{2}+\mathrm{P} \\
\mathrm{v}(\rho \mathrm{E}+\mathrm{P}) \\
\rho \mathrm{vY}_{\mathfrak{i}}
\end{array}\right| \\
& \mathrm{F}_{\mathrm{v}}=\left|\begin{array}{l}
0 \\
\tau_{\mathrm{xx}} \\
\tau_{\mathrm{xy}} \\
\mathrm{u} \tau_{\mathrm{xx}}+v \tau_{\mathrm{xy}}-\mathrm{q}_{\mathrm{x}} \\
\rho \tilde{\mathrm{u}}_{\mathrm{i}} \mathrm{Y}_{\mathrm{i}}
\end{array}\right|, \quad \mathrm{G}_{\mathrm{v}}=\left|\begin{array}{l}
0 \\
\tau_{\mathrm{xy}} \\
\tau_{\mathrm{yy}} \\
\mathrm{u} \tilde{\mathrm{\tau}}_{\mathrm{xy}}+\mathrm{v} \tau_{\mathrm{yy}}-\mathrm{q}_{\mathrm{y}} \\
-\rho \tilde{v}_{\mathbf{i}} \mathrm{Y}_{\mathrm{i}}
\end{array}\right| \\
& S=\left|\begin{array}{c}
0 \\
0 \\
0 \\
0 \\
\dot{w}_{i}
\end{array}\right|, i=1,2, \ldots, N_{S}-1
\end{aligned}
$$




$$
\text { and } \begin{aligned}
\tau_{\mathrm{xx}} & =2 \mu \mathrm{u}_{\mathrm{x}}-(2 / 3) \mu\left(\mathrm{u}_{\mathrm{x}}+\mathrm{v}_{\mathrm{y}}\right) \\
\tau_{\mathrm{xy}} & =\mu\left(\mathrm{u}_{\mathrm{y}}+\mathrm{v}_{\mathrm{x}}\right) \\
\tau_{\mathrm{yy}} & =2 \mu \mathrm{v} y-(2 / 3) \mu\left(\mathrm{u}_{\mathrm{x}}+\mathrm{v}_{\mathrm{y}}\right) \\
\mathrm{q}_{\mathrm{x}} & =-\mathrm{k} \frac{\partial \mathrm{T}}{\partial \mathrm{x}}+\rho \sum_{i=1}^{\mathrm{N}_{\mathrm{S}}} \mathrm{h}_{\mathbf{i}} \mathrm{T}_{\mathbf{i}} \tilde{\mathrm{u}}_{\mathrm{i}} \\
\mathrm{q}_{\mathrm{y}} & =-\mathrm{k} \frac{\partial \mathrm{T}}{\partial \mathrm{y}}+\rho \sum_{\mathrm{i}=1}^{\mathrm{N}_{\mathrm{S}}} \mathrm{h}_{\dot{i}} \mathrm{~T}_{\mathrm{i}} \tilde{\mathrm{v}}_{\mathbf{i}}
\end{aligned}
$$

The diffusion velocities are found by fick's law

$$
\begin{aligned}
& \mathrm{Y}_{i} \tilde{u}_{i}=-D_{i m} \frac{\partial Y_{i}}{\partial x} \\
& Y_{i} \tilde{v}_{i}=-D_{i m} \frac{\partial Y_{i}}{\partial y} \\
& D_{i m}=\left(1-X_{i}\right) / \sum_{j \neq i}\left(X_{j} / D_{i j}\right)
\end{aligned}
$$

where $D_{i m}$ is the binary diffusivity for species $i$ in the gas mixture. The temperature and pressure are calculated iteratively from the following equations:

$$
\begin{aligned}
& E=\sum_{i=1}^{N_{S}} h_{i} Y_{i}-\frac{p}{\rho}+\frac{u^{2}+v^{2}}{2} \\
& h_{i}=h_{i}^{0}+\int{ }_{T_{r}}^{T} C_{i} d T \\
& p=\rho \tilde{R T} \sum_{i=1}^{N_{S}^{S}}\left(Y_{i} / M_{i}\right)
\end{aligned}
$$

Here $Y_{j}, X_{i}$ and $M_{j}$ are the mass fraction, molar fraction and molecular weight for species i.

\section{THERMODYNAMIC AND TRANSPORT MODELS}

The specific heat, thermal conductivity and viscosity for each species are determined by fourth-order polynominals of temperature, such as:

$$
C_{p_{i}}=A_{i}+B_{i} T+C_{i} T^{2}+D_{i} T^{3}+E_{i} T^{4}
$$


The coefficients of these polynominals are supplied by McBride (ref. 4) and are valid up to a temperature of $6000 \mathrm{~K}$. The specific heat of the gas mixture is obtained by concentration weighting of each species. The thermal conductivity and viscosity of the mixture, however, are calculated using Wilke's mixing rule (ref. 5).

The binary mass diffusivity $D_{j} i$ between species $i$ and $j$ is obtained using the Chapman-Enskog theory in conjuction with the Lennard-Jones intermolecular potential-energy functions (ref. 5). The diffusion of a species in the gas mixture is approximated by Fick's Law, i.e., treating the species $i$ and the surrounding gas as a binary gas mixture, and the diffusion velocity for each species is calculated using equation (2).

\section{CHEMISTRY MODELS}

In the present study, the finite-rate reaction of hydrogen and air are considered for the chemistry model. The reaction model of Brabbs (ref. 6) is used. This model employs 9 species and 18 elementary reactions. Nitrogen is treated as an inert species. Nitrogen was not specified for the thruster calculation. For a set of $N_{R}$ elementary reactions involving $N$ species, the rate equations can be written in the following general form:

$$
\sum_{j=1}^{N_{s}} \gamma_{n j}^{\prime} \quad C_{j} \quad \stackrel{k_{f}}{\underset{k_{b_{i}}}{\rightleftharpoons}} \quad \sum_{j=1}^{N_{s}} \gamma_{n j}^{\prime \prime} C_{j}, i=1,2, \ldots, N_{R}
$$

Here $C_{j}$ is the molar concentration of the $j$ th species. The forward reaction rate constant $k_{f}$ for the $i^{\text {th }}$ elementary reaction is given by the Arrhenius equation:

$$
k_{f} \quad=A_{i} T^{\mathrm{B}_{i}} \exp \left(-E_{i} / R T\right)
$$

where $E_{\mathfrak{i}}$ represents the activation energy, and $A_{\mathfrak{j}}$ and $B_{\mathfrak{i}}$ are constants. The backward reaction rate constant is calculated using the forward reaction rate constant and the equilibrium coefficient $k_{i}$,

$$
k_{b_{i}}=k_{f_{i}} / k_{i}
$$

The rate of change of molar concentration of species $j$ by reaction $i$ is given by

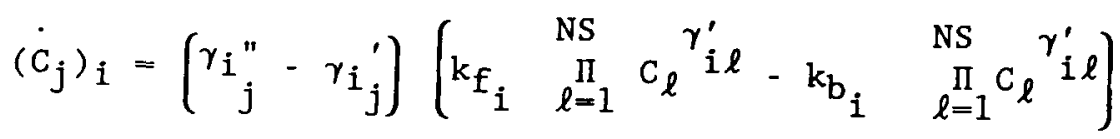


The total rate of change of molar concentration of species $j$ is then given by

$$
\dot{C}_{j}=\sum_{i=1}^{N_{R}}\left(\dot{C}_{j}\right)_{i}
$$

the chemical source terms can be found from

$$
\dot{W}_{j}=\dot{C}_{j} M_{j}
$$

\section{TURBULENCE MODEL}

The zero-equation algebraic turbulence model developed by Baldwin-Lomax (ref. 7) is used. This model is a two-layer model in which an eddy viscosity is calculated for an inner and outer region. In both of the regions the distribution of vorticity is used to determine the length scales. The primary advantage of this model is its simplicity and that boundary layer thickness does not need to be calculated.

\section{NUMERICAL TECHNIQUE}

Many numerical techniques have been used to solve the set of equations governing chemically reacting flows. Among these techniques, the explicit schemes are generally slow in convergence when the flow involves high rates of heat release or zones of recirculation. Most implicit schemes, on the other hand, require the inversion of block matrices and become exceedingly expensive when the chemical system involves a large number of species. In the present study, the lower-upper symmetric overrelaxation (LU-SSOR) scheme of Yoon and Jameson 2 is adopted to solve the two-dimensional Navier-Stokes and species transport equations. The LU-SSOR scheme employs implicit Newton iteration technique to solve the finite-volume approximation of the steady-state version of the governing equations. The convergence of the Newton iteration method is assured by the diagonal dominance of the coefficient matrices of the LU-SSOR scheme. The detailed derivations of the LU-SSOR scheme for nonreacting and reacting flows can be found in references 2 and 3 , repsectively. The final discretized form of the equations for chemically reacting flows are given by

$$
\begin{gathered}
\left(D_{x}{ }^{-} A^{+}+D_{y}{ }^{-} B^{+}-A^{-}-B^{-}-T\right)\left(D_{x}{ }^{+} A^{-}+D_{y}{ }^{+} B^{-}+A^{+}+B^{+}\right) \delta W \\
=-\left(\gamma_{A}+\gamma_{B}\right)\left[D_{x}\left(F-F_{v}\right)^{n}+D_{y}\left(G-G_{v}\right)^{n}-S^{n}\right]
\end{gathered}
$$

where the Jacoabian matrices $A, B$ and $T$ are defined by 


$$
\begin{aligned}
& A=\frac{\partial F}{\partial W} \\
& B=\frac{\partial G}{\partial W} \\
& T=\frac{\partial S}{\partial W}
\end{aligned}
$$

Here because of the stiffness of the equations for reacting flows, the chemical source terms have been treated implicitly. Bussing and Murman (ref. 8), and Eklund et al. (ref. 9) have shown that implicit treatment of the chemical source terms is equivalent to rescaling the governing equations in time and thus stablizes the numerical scheme.

In equation (12), $D_{x}^{-}$and $D_{y}^{-}$are backward difference operators and $D_{x}{ }^{+}$ and $\mathrm{Dy}^{+}$are forward difference operators. $\mathrm{A}^{+}, \mathrm{A}^{-}, \mathrm{B}^{+}$and $\mathrm{B}^{-}$are constructed so that the eigenvalues of "+" matrices are nonnegative and those of "-" matrices are nonpositive, as follows:

$$
\begin{aligned}
& \mathrm{A}^{+}=1 / 2\left(\mathrm{~A}+\gamma_{\mathrm{A}} \mathrm{I}\right) \\
& \mathrm{A}^{-}=1 / 2\left(\mathrm{~A}-\gamma_{\mathrm{A}} \mathrm{I}\right) \\
& \mathrm{B}^{+}=1 / 2\left(\mathrm{~B}+\gamma_{\mathrm{B}} \mathrm{I}\right) \\
& \mathrm{B}^{-}=1 / 2\left(\mathrm{~B}-\gamma_{\mathrm{B}} \mathrm{I}\right)
\end{aligned}
$$

where

$$
\begin{aligned}
& \gamma_{A} \geq \max \left(\left|\lambda_{A}\right|\right) \\
& \gamma_{B} \geq \max \left(\left|\lambda_{B}\right|\right)
\end{aligned}
$$

Here, $\lambda_{A}$ and $\lambda_{B}$ represent eignevalues of Jacobian matrices. becomes

After expanding out the difference operators on the LHS, equation (12)

$$
\begin{gathered}
\left(\gamma_{A} I+\gamma_{B} I-A_{i-1, j}^{+}-B_{i, j-1}^{+}-T_{i, j}\right)\left(\gamma_{A} I+\gamma_{B} I+A_{i+1, j}^{-}-B_{i, j+1}^{-}\right) \\
=-\left(\gamma_{A}+\gamma_{B}\right)\left[D_{x}\left(F-F_{v}\right)^{n}+D_{y}\left(G-G_{v}\right)^{n}-s^{n}\right]
\end{gathered}
$$

It is interesting to note that for nonreacting flows ( $S=0$ and $T=0$ in eq. (16)) the present numerical method eliminates the need for banded block matrix inversions without using a diagonalization procedure. In fact, with a forward diagonal sweep for the inversion of the first operator and a backward diagonal sweep for the inversion of the second operator, only scalar diagonal inversions are needed to solve equation (16) for nonreacting flow problems.

For reacting flows, due to the presence of the chemical source Jacobian $T$, the first operator on the left-hand side of equation (16) now requires block diagonal inversions. However, since in the present formulation the flow equations (continuity, momentum, and energy equations) have no source terms, 
the first four rows of the diagonal block of this operator have nonzero terms only in the diagonal. As a result, the first operator of equation (16) can be inverted in essentially two separate steps: the scalar diagonal matrix inversion for the flow equations and the block diagonal matrix inversion for the species equations. In contrast, most other implicit schemes require block banded (tridiagonal or pentadiagonal) matrix inversions for both operators for either the reacting or nonreacting flows. Here, two advantages in efficiency are obtained by the LU-SSOR scheme over other implicit schemes: (a) the block size is smaller in the LU-SSOR scheme. Since the operational count is proportional to $\mathrm{N}^{3}$ for inverting a $\mathrm{N} \times \mathrm{N}$ matrix, the saving in CPU time can be significant; (b) the operational count for inverting a block diagonal matrix is only a fraction of that for inverting a block banded matrix.

Another important efficiency advantage of the LU-SSOR scheme is the vectorizability of its implicit operators. For example, the inversion of the two LHS operators of equations (16) can be fully vectorized if the solution sweeps are taken along the diagonal directions (the directions that $i+j$ increases or decreases). It is noted that the vectorizability is rather unique to the LU scheme, and, few other implicit scheme can have the LHS operators fully vectorized. The vectorizability of the LHS operators is particularly significant for reacting flow calculations, since the most time-consuming operation of inverting the chemical source Jacobian matrix can then be executed simultaneously for a large number of points on the same vector plane, resulting in a large saving of CPU time.

\section{GEOMETRY AND BOUNDARY CONDITIONS}

A unique subsonic inlet condition procedure for chemically reacting flows has been developed in this study for the convergent-divergent nozzle calculations. As total mass flow through the thruster is determined by conditions at the thruster-throat, the inlet velocitites could not be specified. The total enthalpy and mass fractions are specified at the inlet and velocity is extrapolated from the interior. The pressure is obtained using an isentropic relationship.

The subsonic inflow boundary condition procedure is briefly outlined in the following.

Step 1:

The isentropic assumption at the inflow gives

$$
\mathrm{dh}=\frac{\mathrm{dp}}{\rho}
$$

which can be written as

$$
\frac{C_{p} d T}{T}=R \frac{d p}{P}
$$


By assuming frozen species composition, the gas constant, $R$, is a constant, and thus equation (18) can be integrated to yield a relationship between temperature, $T$, and pressure, $p$, at the inflow,

$$
\int_{T_{\text {ref }}}^{T} \frac{C_{p} d T}{T}=R \ln \frac{p}{P_{\text {ref }}}
$$

In equation (19) the polynominal function of $T$ for $C_{p}$ is ultilized to obtain an explicit form relating $T$ and $p$.

\section{Step 2:}

Step $v=0$, and extrapolate $u$ from interior. With known total enthalpy, the specific enthalpy can then be calculated.

\section{Step 3:}

Using equations (4) and (19), temperature and pressure are obtained, and density is then calculated from the equation of state.

For the test problems considered here, the outflows are always supersonic, hence, the dependent variables are extrapolated from the interior. Along the solid wall, no-slip conditions are specified. The wall is assumed adiabatic. Along a plane of symmetry, the normal derivative of all dependent variables are zero, except for $v$ velocity, where $v=0$ is specified.

The configuration used for the small thruster simulation is the downsized rocket engine design with a large expansion nozzle. A schematic is shown in figure 1. Oxygen and hydrogen are used for propellants. A surplus of hydrogen was used for wall cooling as well as greater thrust. The thruster inlet has two inlet zones. The core flow consists of gaseous hydrogen and oxygen at an equivalence ratio of 0.5 . The second inlet flow is pure hydrogen used for direct wall cooling. Approximately, 5 percent of the outer inlet area is used for the second inlet flow. The two inlet flows are separated by a cylindrical sleeve with milled slots for the hydrogen. The sleeve and combustion within the sleeve are not modeled in this calculation, instead, the calculation starts at the point past the sleeve. In the actual thruster combustion is initiated by an igniter. The igniter is only used momentarily as the reaction is self sustaining. The inlet is rather warm as the propellants were initially used for wall cooling. This causes rather high temperatures for the combustion products in the core zone. In the calculation the core flow is modeled by a $3000 \mathrm{~K}$ mixture of oxygen and combustion products (table I). The inlet conditions came from a sequence of initial runs. The first calculation used equilibirium mixture. This mixture then changed due to the use of a finite rate chemistry model in the code. Mixture fraction values along the forward centerline were used for successive calculations. A 1.5 in. long combustion chamber is provided where the oxygen rich core flow and wall cooling hydrogen can react. The flow is then accelerated through a convergingdiverging nozzle. The expansion ratio used in the actual thruster was around 
100. For the present two-dimensional rectangular calculation an area expansion ratio of 4.2 is used. The grid for the calculation is shown in figure 2 . The grid is finer near the walls for improved calculation of viscous effects and the mixing and chemical reactions of the shear layer.

A second calculation is also presented. This involves a large area-ratio expansion nozzle. The area ratio is approximately 20:1 and is generated by a partial sine curve. The inlet Mach number of 1.1 is specified. The temperature and species concentrations are similar to the core flow for the thruster. The species concentrations were generated using the equilibium code of Gordon and MCBride (ref. 12). An inert fraction of 80 percent nitrogen was specified. The inlet species fractions are listed in table I.

\section{RESULTS AND DISCUSSION}

\section{Small thruster}

A contour plot of Mach number for the small thruster calculation is shown in figure 3. Mach number increases along fluid flowpath. Mach number contours are remarkably similar to other calculations of this thruster geometry, whether they involve reaction or not. The maximum Mach number is 2.699 and occurs along the exit centerline. Approximately $2 / 3$ of the way through the converging part of the nozzle the maximum Mach number (at a fixed axial distance) changes from occurring along the centerline to approximately. 85 percent of the transverse distance to the thruster wall. This increase in Mach number at this location is caused by the reduction in flow area especially for the outer flow.

A contour plot of the pressure field is shown in figure 4 . Pressure decreases along fluid flowpath. The highest pressure is found in the area of hydrogen injection. Pressure increases slightly with increasing radial distance in the converging portion of the thruster nozzle. Pressure is lower closer to the thruster wall in the initial expansion. Pressure then becomes slightly higher near the walls where velocity is lowest. The lowest pressure occurs at the exit centerline (the point of maximum Mach number).

Contour plots of $\mathrm{O}_{2}, \mathrm{H}_{2}$, and $\mathrm{H}_{2} \mathrm{O}$ are shown in figures 5 to 7 , respectively. As expected, the oxygen mass fraction decreases towards the thruster wall, due to the lack of mixing in this region. Oxygen concentration does not vary much along the nozzle centerline. The hydrogen mass fraction increases with decreasing distance from the thruster wall. The maximum $\mathrm{H}_{2}$ mass fraction at the exit is 0.97 . The maximum centerline exit $\mathrm{O}_{2}$ mass fraction is 0.455 . Much of the oxygen and hydrogen did not mix, causing a loss in thruster performance. A turbulence model tailored for a reacting shear layer would be more appropriate than the model used for this calculation (better mixing would also be obtained by modeling the turbulence generated by the core flow combustion and the actual inlet geometry). The water mass fraction reaches a transverse maximum where the shear layer is reacting. The maximum water fraction is 0.918 and occurs at the exit of the thruster in figure 7 (the plotting package truncated the list of contour levels).

A contour plot of temperature is shown in figure 8 . Overall temperature drops through the thruster due to expansion and mixing. The maximum radial temperature at the inlet shows up at 0.9 of the total radial distance. The location of maximum temperature at a fixed axial distance moves further away 
from the nozzle wall through the combustion chamber and the converging portion of the nozzle. The maximum radial temperature then occurs along the thruster centerline for the first half of the divergent section of the nozzle. In the final half of the divergent section, centerline thermal energy is more completely changed to kinetic energy and the location of maximum temperature moves away from the centerline. The nozzle wall temperature is much lower than the temperature in the mixing layer and the core flow, indicating that the hydrogen wall jet has well served as cooling protection for the wall.

$\mathrm{OH}$ mass fraction contours are shown in figure 9. The maximum $\mathrm{OH}$ mass fraction occurs near the nozzle wall. This does not occur exactly where the maximum (sometimes local) radial temperature does, but slightly closer to the thruster wall where the hydrogen mass fraction is higher. The location of maximum water concentration is also slightly closer to the thruster wall.

\section{DIVERGING NOZZLE}

The pressure contours are shown in figure 10. Pressure decreases with axial distance. The pressure contours are parabolic in shape. This same shape is shown by the Mach contours in figure 11, except near the wall, where viscous effects predominate. The maximum Mach number for the diverging nozzle is 4.103 .

Temperature contours are shown in figure 12. The location of maximum temperature is at the nozzle wall, due to viscous heating. In the core flow region, temperture initially rises slightly. This is attributed to recombination reactions, which release thermal energy to the flow. It is noted that the initial large amount of recombination is partly due to the mismatch of the equilibrium composition specified at the inflow and the finite-rate chemistry model.

In figure $13, \mathrm{H}_{2} \mathrm{O}$ contours show a slight decrease at the inlet. This is due to the change of temperature and pressure caused by the flow expansion, and the change in chemical reaction models, upsetting the inlet equilibrium composition. Further downstream from the inlet, the chemical reactions slow down and gradually reach the frozen chemistry condition, as evidenced by the contour plots. The slowing down of chemical reactions is caused by the decrease in temperature and pressure due to expansion. Most of the change in water mass fraction with radial distance occurs near the nozzle wall, due to the large temperature change near the wall. The hydrogen, oxygen and hydroxol concentrations shown in figures 14 to 16 , follow temperature contours very closely. Oxygen mass fraction initially increases along axial direction, due to recombination reactions. Hydroxol mass fraction initially drops to 50 percent of inlet values and then further decreases with decreasing temperature. Oxygen mass fraction increases with decreasing temperature, except for a small initial change. Monatomic oxygen mass fraction, shown in figure 17 , increases rapidly near the inlet showing a mismatch in the equilibrium inlet condition and the finite-rate chemistry model in the code. Near the nozzle wall, monatomic oxygen mass fraction increases, due to the dissociation reactions caused by higher temperatures in this region. Monatomic oxygen does decrease along the nozzle centerline after the adjustment in chemistry models, due to recombination reactions. 


\section{CONCLUDING REMARKS}

The flowfields in converging-diverging ducts and supersonic nozzles have been analyzed using a comprehensive reacting flow code. The calculations show the hydrogen wall cooling to be effective for an initial hydrogen-rich thruster design. A calculation for a diverging nozzle also shows the code to be capable of calculating reacting nozzle flow where the change in area ratio is large. The results indicate that the qualititative features of the flowfield and chemical reactions are well represented by the calculations. However, mixing between the wall cooling $\mathrm{H}_{2}$ jet and the hot gas in the core region for the small thruster is underpredicted. A turbulence model more sophisticated than the Bladwin-Lomax model used in the present study may be required to correctly predict the mixing behavior of the flow.

\section{REFERENCES}

1. Jameson, A. and Turkel, E., "Implicit Schemes and LU Decompositions, "Mathematics of Computation, Vol. 37, No. 156, 1981, pp. 385-397.

2. Yoon, S. and Jameson, A., "A LU-SOOR Scheme for the Euler and Navier-Stokes Equations," AIAA Paper 87-0600, Jan. 1987.

3. Shuen, J.S., and Yoon S., "Numerical Study of Chemically Reacting Flows Using a LU Scheme," Paper 88-0436, Jan. 1988 (to appear in AIAA J.).

4. MCBride, B.J., Private Communication, NASA Lewis Research Center, Cleveland, $\mathrm{OH}, \mathrm{Apr} .1987$.

5. Reid, R.C., Prausnitz, J.M., and Sherwood, T.K., The Properties of Gases and Liquids. Third edition, McGraw-Hill Publishing Co., New York, 1977.

6. Brabbs, T.A., Private Communication, NASA Lewis Research Center, Cleveland, $\mathrm{OH}, 1988$.

7. Baldwin, B. and Lomax, H., "Thin Layer Approximation and Algebraic Model for Separated Turbulenet Flows," AIAA Paper. 78-257, Jan, 1978.

8. Bussing, T.R. and Murman, E.M., "A Finite Volume Method for the Calculation of Compressible Chemically Reacting Flows," AIAA Journal, Vol. 26, No. 9, Sept. 1988, pp. 1070-1078.

9. Eklund, D.R., Hassan, H.A., and Drummond, J.P., "The Efficient Calculation of Chemically Reacting Flow," AIAA Paper 86-0563, Jan. 1986. 
TABLE I. - THRUSTER AND DIVERGING NOZZLE INLET MASS FRACTIONS

\begin{tabular}{|c|c|c|c|c|c|c|c|c|c|}
\hline \multirow{2}{*}{ Zone } & $\mathrm{H}_{2}$ & $\mathrm{O}_{2}$ & $\mathrm{OH}$ & $\mathrm{H}_{2} \mathrm{O}$ & $\mathrm{H}$ & $\mathrm{O}$ & $\mathrm{HO}_{2}$ & $\mathrm{H}_{2} \mathrm{O}_{2}$ & $\mathrm{~N}_{2}$ \\
\cline { 2 - 9 } & \multicolumn{8}{|c|}{ Thruster mass fractions } \\
\hline 1 & $\begin{array}{l}0.001 \\
1.0\end{array}$ & $\begin{array}{l}0.438 \\
0.0\end{array}$ & $\begin{array}{l}0.029 \\
0.0\end{array}$ & $\begin{array}{l}0.504 \\
0.0\end{array}$ & $\begin{array}{l}0.0 \\
0.0\end{array}$ & $\begin{array}{l}0.028 \\
0.0\end{array}$ & $\begin{array}{l}0.0 \\
0.0\end{array}$ & $\begin{array}{l}0.0 \\
0.0\end{array}$ & $\begin{array}{l}0.0 \\
0.0\end{array}$ \\
\cline { 2 - 9 } & \multicolumn{8}{|c|}{ Diverging nozzle mass fractions } \\
\cline { 2 - 10 } & 0.001 & 0.078 & 0.020 & 0.094 & 0.0 & 0.007 & 0.0 & 0.0 & 0.8 \\
\hline
\end{tabular}

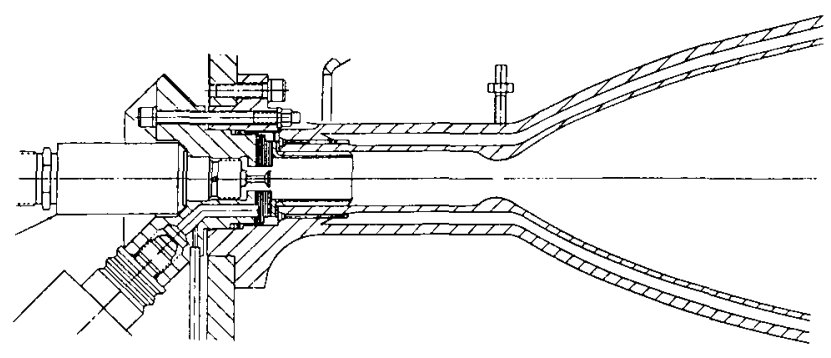

figuRE 1. - initial SPACE-STATION THRUSTER DESIGN.

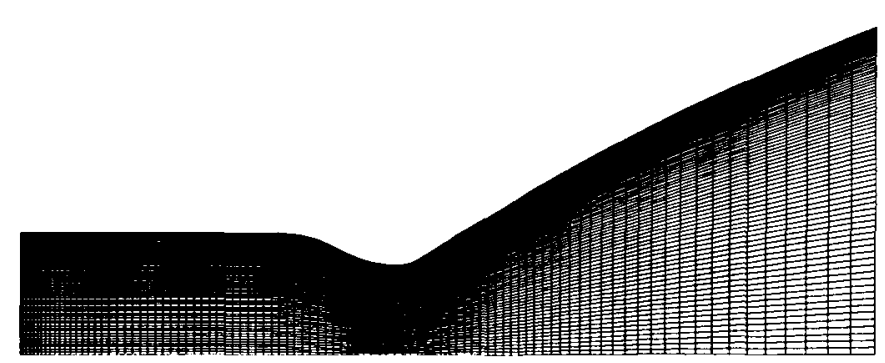

FIGURE 2. - THRUSTER CALCULATIONS GRID. 
CONTOUR LEVELS

0.00000

0.10000

0.20000

0.30000

0.40000

0.50000
0.60000

0.70000

0.80000

0.90000

1. 00000

1. 10000

1. 20000

1.30000

1. 40000

1.50000

1.60000

1. 70000

1. 80000

1.90000

2. 00000

2. 10000

2.20000

2.30000

2.40000

2.50000

2. 60000

2. 70000

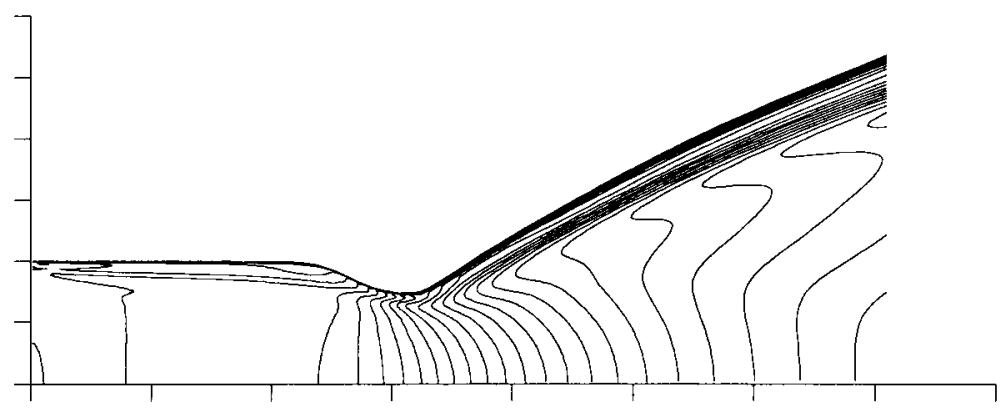

FIGURE 3. - MACH NUMBER CONTOURS.

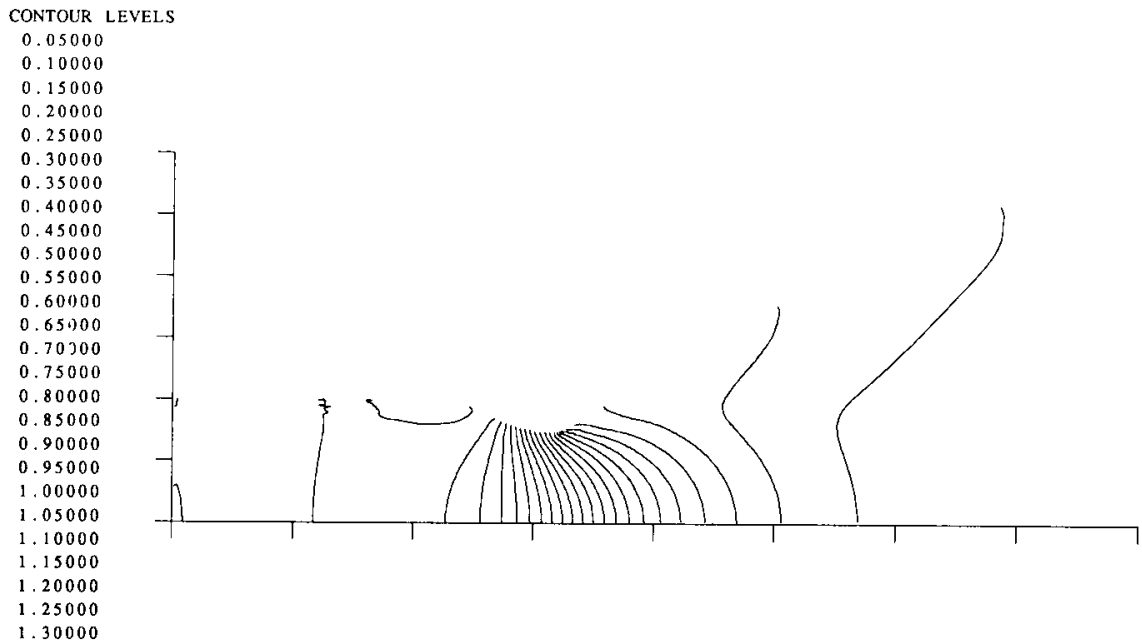

FIGURE 4. - PRESSURE CONTOURS.

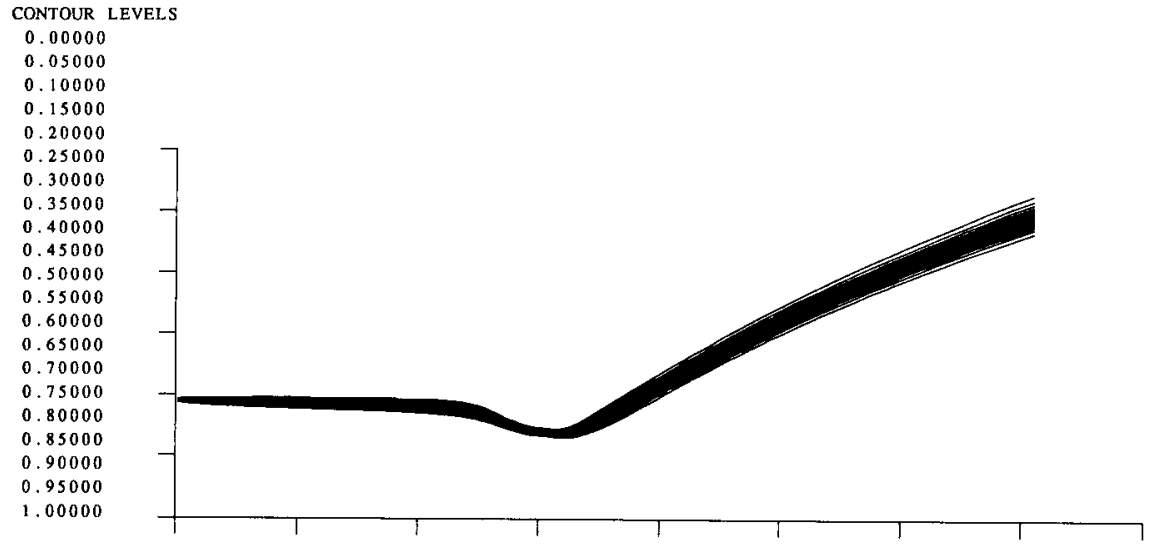

FIGURE 5. - OXYGEN MASS FRACTION CONTOURS. 


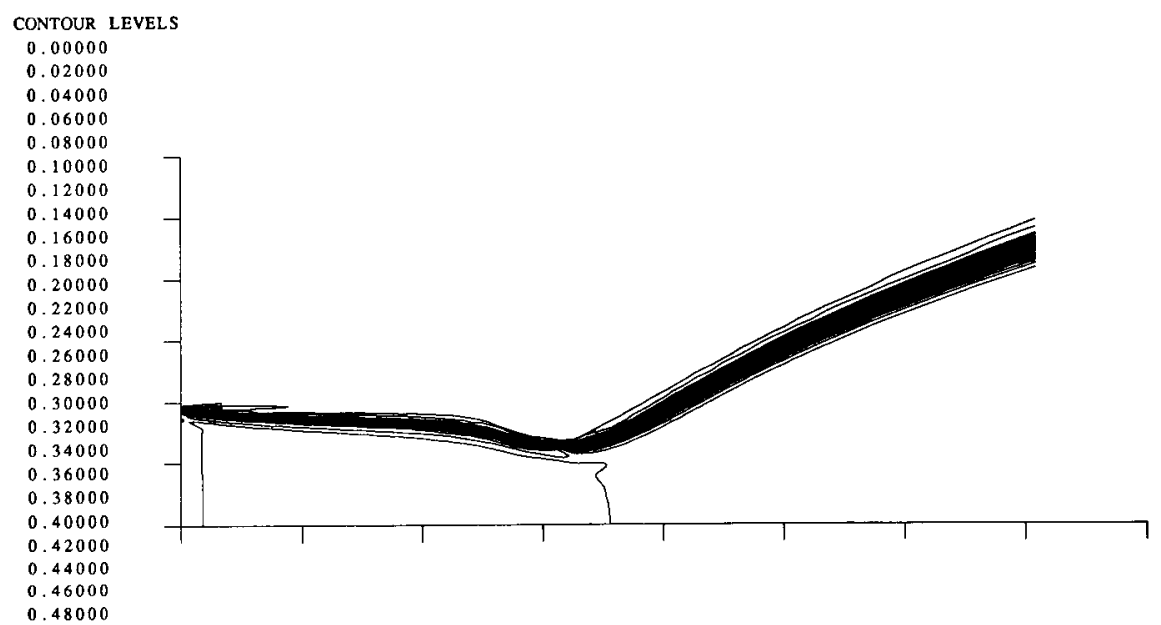

FIGURE 6. - HYDROGEN MASS FRACTION CONTOURS.

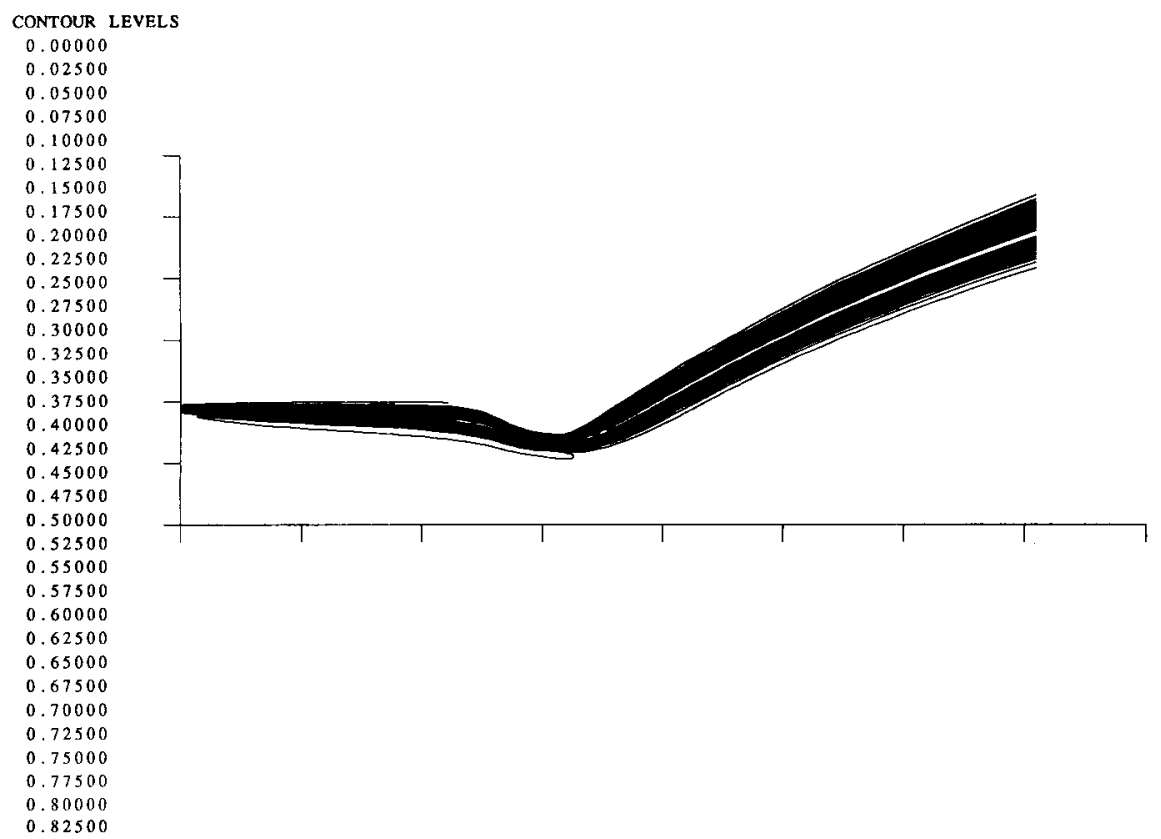

FIGURE 7. - WATER MASS FRACTION CONTOURS. 




FIGURE 8. - TEMPERATURE COWTOURS.

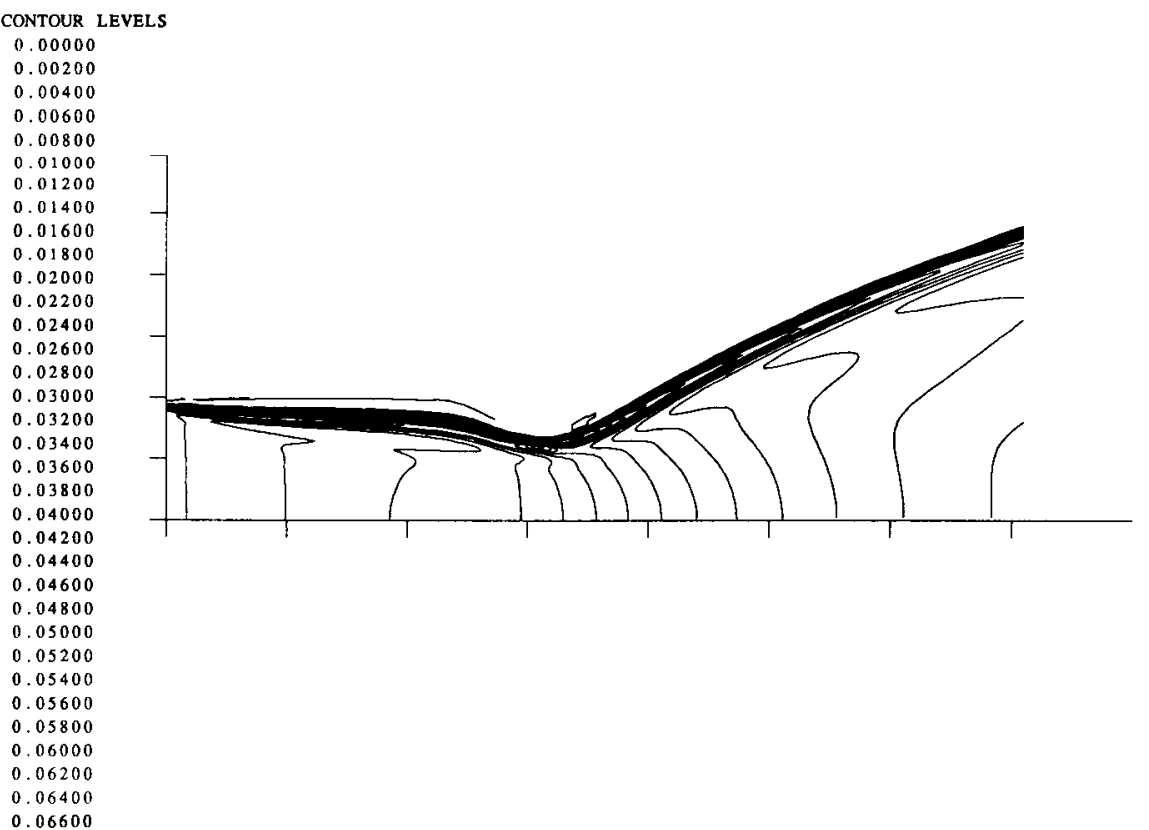

FIGURE 9. - HYDOXO MSS FRACTION CONTOURS. 


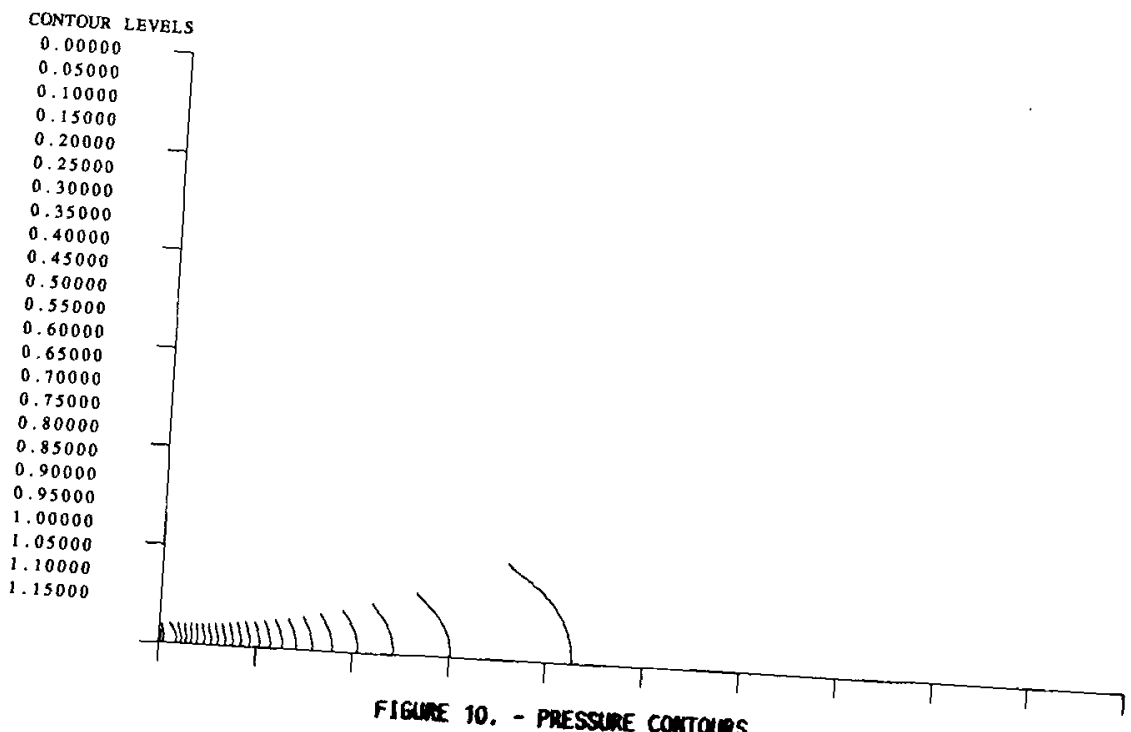

FIGLine 10. - PREsstie comous.

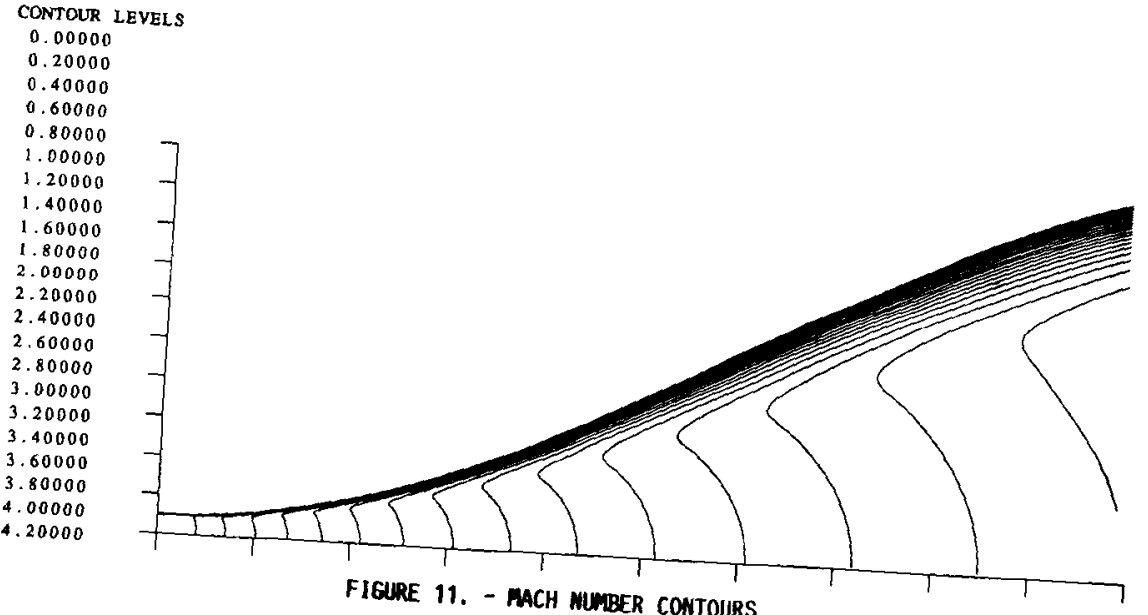

GURE 11. - MACH MUMBER CONTOURS.

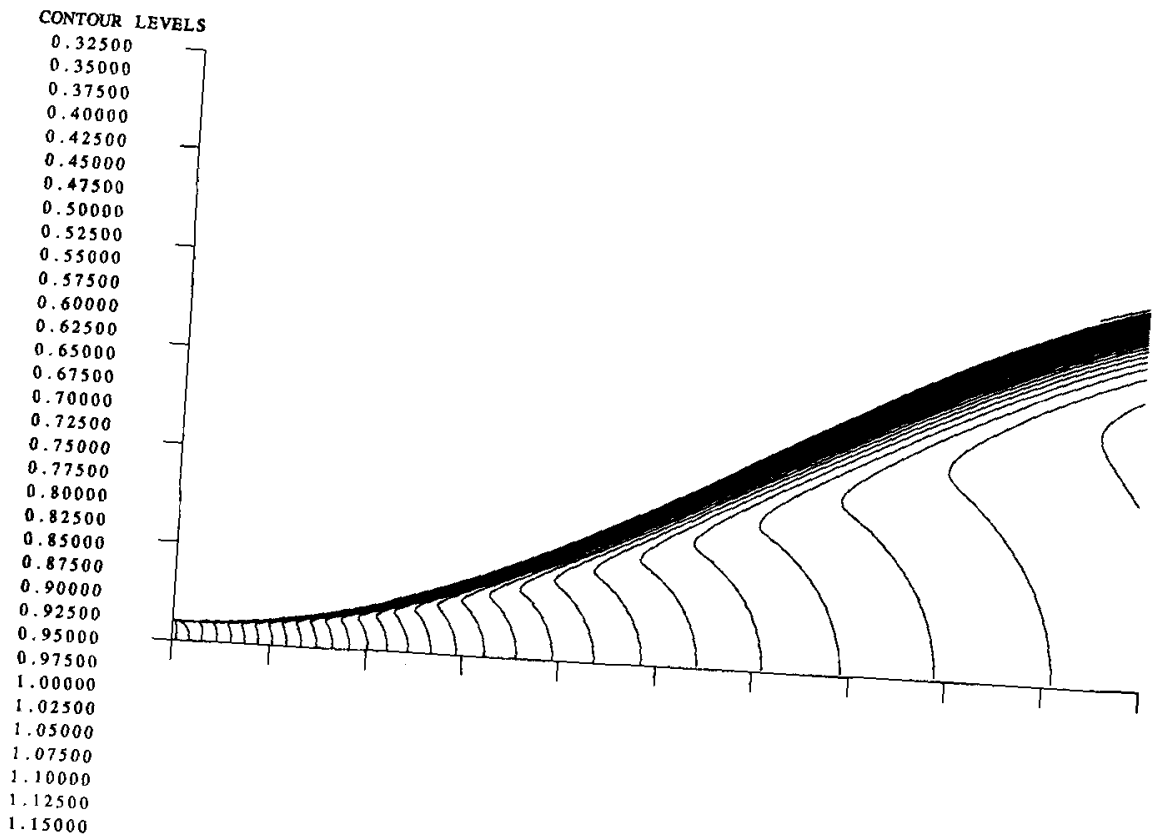

FIGURE 12. - TEMPERATURE CONTOURS. 


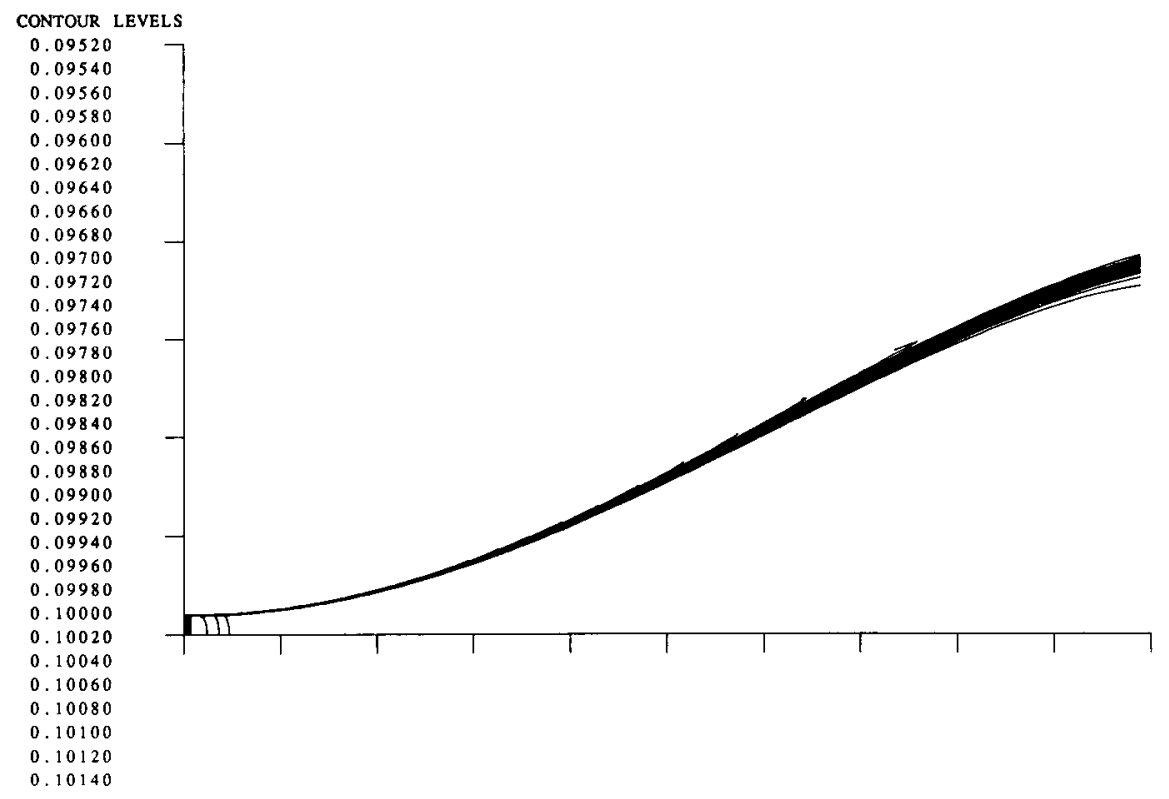

FIGURE 13. - WATER MASS FRACTION CONTOURS.

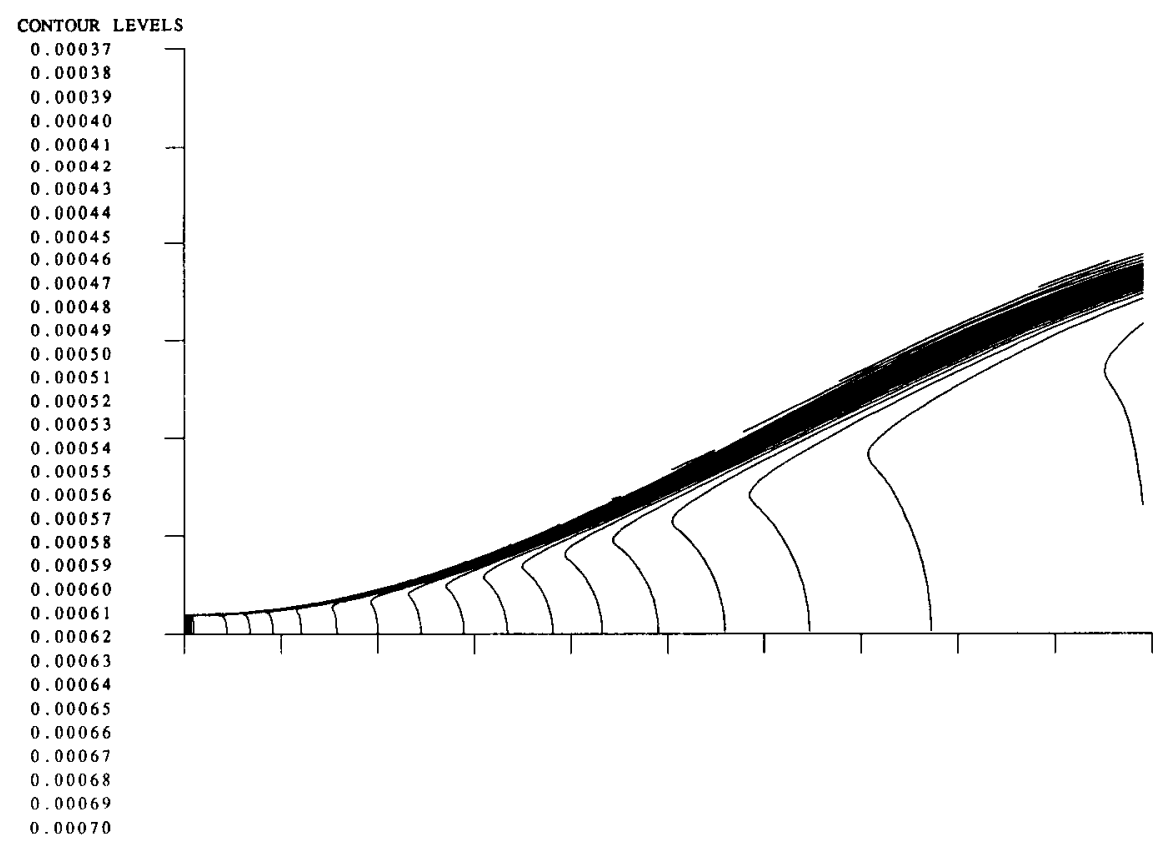

FIGURE 14. - HYDROGEN MASS FRACTION COMTOURS. 


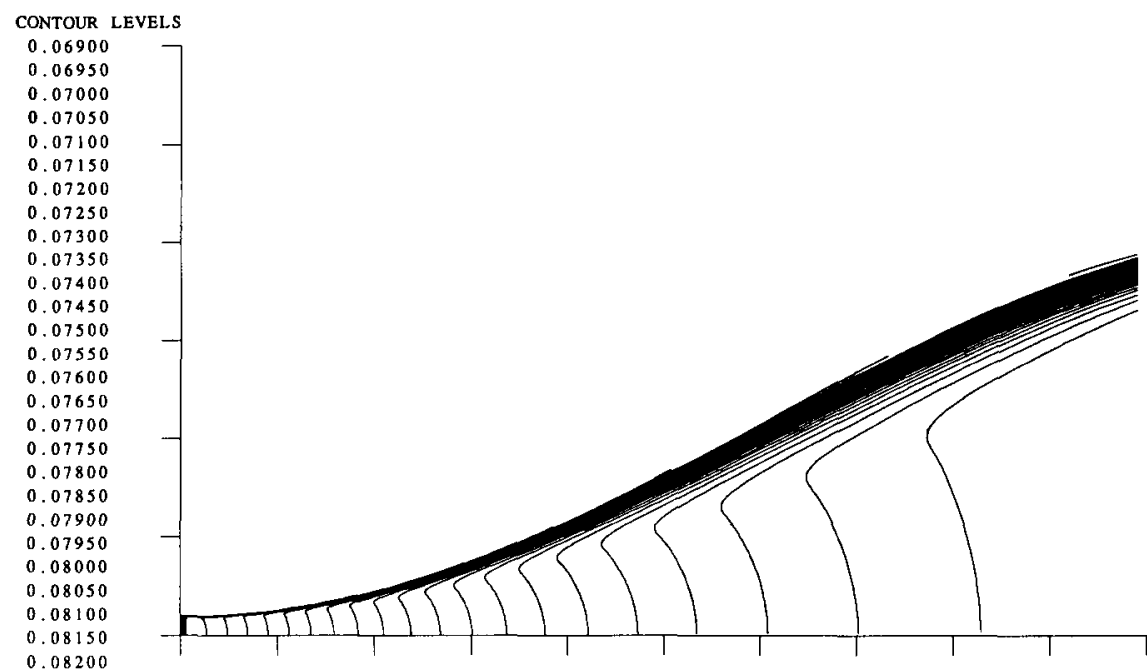

0.08200

0.08250

0.08300

0.08350

0.08400

0.08450

FIGURE 15. - OXYGRN MASS FRACIION CONTOURS.

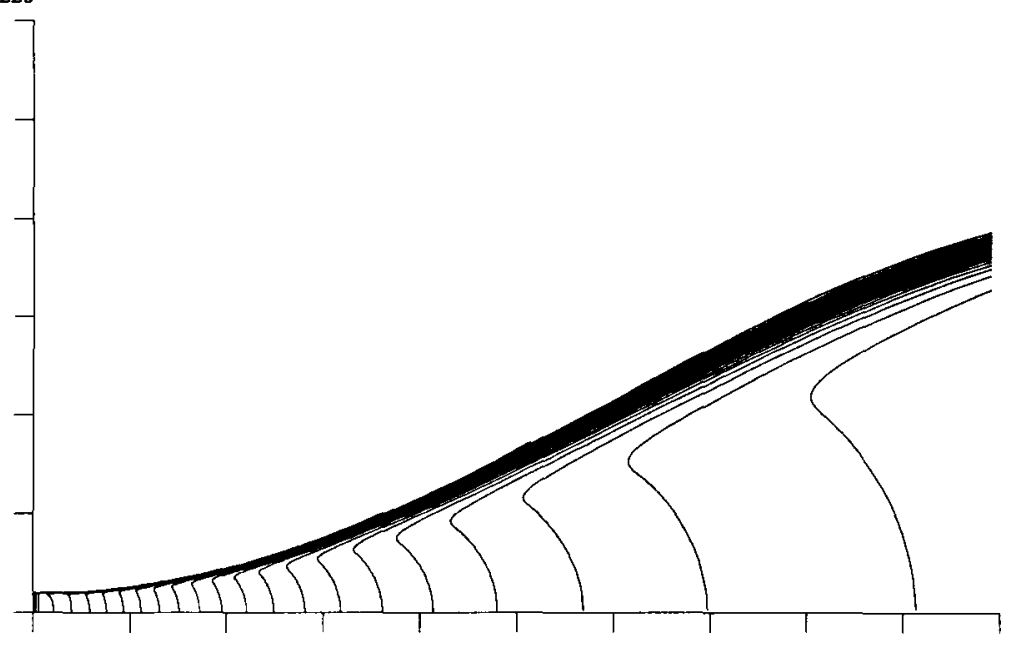

FIGURE 16. - HYDROXOL MASS FRACTION CONTOURS. 


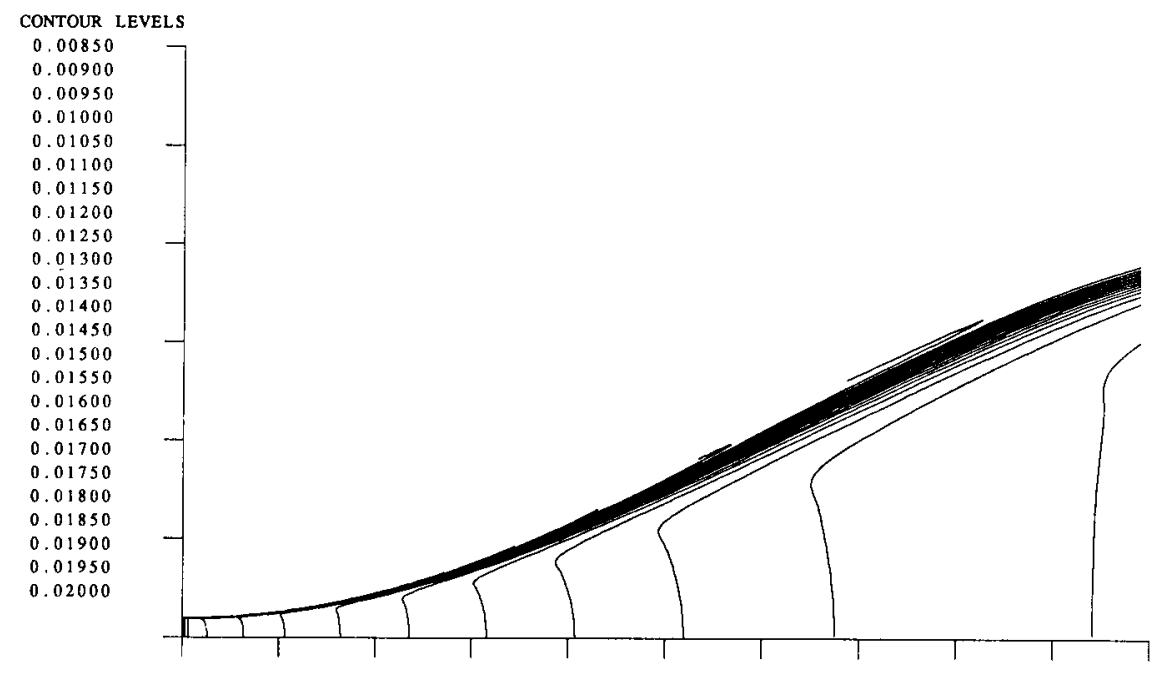

FIGURE 17. - MONATONIC OXYGEN MASS FRACTION CONTOURS. 




